\title{
Estudio correlacional entre la modalidad de educación pública o privada y el estilo de aprendizaje
}

\section{Correlational study education mode public or private and style of learning}

\author{
Gerson Adriano Rincón-Álvarez¹, Daniel Villamizar-Jaimes², Carlos Antonio Pabón-Galán³
}

Forma de citar: G. A. Rincón, D. Villamizar, C. A. Pabón. Estudio correlacional entre la modalidad de educación pública o privada y el estilo de aprendizaje. Eco.Mat. 2015; 6(1): 69-77.

Recibido:

Noviembre 22

de 2014

Aceptado:

Diciembre 18 de

2014

${ }^{1}$ Universidad Francisco de Paula Santander, gersonadrianora@ufps.edu.co

${ }^{2}$ Universidad Francisco de Paula Santander, dvillami@bari.ufps.edu.co

${ }^{3}$ Universidad Francisco de Paula Santander, carlosantoniopg@ufps.edu.co

\section{Resumen}

Siempre ha existido una gran discusión sobre, la diferencia entre la educación pública y privada, referente a muchos tópicos de enseñanza aprendizaje como: la metodología, la filosofía, la preparación de los estudiantes, y en lo referente a este artículo, las diferentes estrategias que emplean los estudiantes para adquirir y fortalecer sus aprendizaje. La identificación de los estilos de aprendizaje y el cómo se desenvuelve el estudiante en el medio universitario es de gran importancia para la adecuación de las prácticas pedagógicas del docente y la construcción de los micro-currículos de las asignaturas, que conforman el pensum académico del programa. Con el fin de generar un mejor ambiente de aprendizaje, esta investigación identifica los estilos de aprendizaje de los estudiantes del programa académico de Licenciatura en Matemáticas de la Facultad de Educación, Artes y Humanidades de la UFPS, enmarcado en la teoría del modelo de Kold. Se concluye que el estilo predominantes en la población objeto de estudio fue el reflexivo seguido del pragmático y por último el activo y el teórico respectivamente. Se finaliza con la aplicación de las pruebas de independencia y correlación de Spearman, hallando alta relación entre los estilos reflexivo y pragmático dentro del modelo de Kold, pero ninguna relación entre el estrato social y los estilos de aprendizaje.

Palabras claves: estilos de aprendizaje, modelo, pensum, micro-currículo, Practica Pedagógica

\section{Summary}

There has always been a big discussion about the difference between public and private education regarding many topics of learning as the methodology, philosophy, preparing students, and in relation to this article, the different strategies employ students to acquire and strengthen their learning. The identification of learning styles and how the student develops in the university environment is of great importance to the adequacy of the pedagogical practices of teachers and the construction of micro-curriculum subjects that make up the academic curriculum of the program. In order to create a better learning environment, this research identifies learning styles of students of the academic Bachelor of Mathematics, Faculty of Education, Arts and Humanities UFPS, framed in the theory of model Kold. We conclude that the predominant style in the study population was followed by the pragmatic and thoughtful finally assets and theoretical respectively. It ends with the application of the tests of independence and correlation of Spearman, finding high relationship between pragmatic and thoughtful styles within the model Kold, but no relationship between social class and learning styles.

Keywords: learning styles, model, curriculum, micro-curriculum Pedagogical Practice 


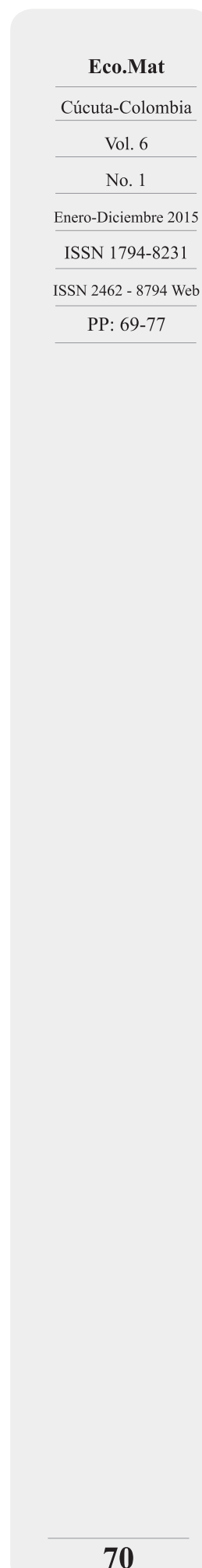

70

Estudio correlacional entre la modalidad de educación pública o privada y el estilo de aprendizaje

\section{INTRODUCCIÓN}

Las últimas investigaciones en las ramas como la neuropsicología, la neurofisiología y en la psicología han dado como resultado nuevos enfoque sobre cómo los seres humanos aprenden, estos estudios indican que cada persona tiene una forma o estilo particular de establecer relación con el mundo y por lo tanto para aprender. Con respecto a este enfoque, se han desarrollado distintos modelos que aproximan una clasificación de estas distintas formas de aprender.

Dentro del marco educativo es necesario e imperativo identificar los estilos de aprendizaje de los estudiantes con el fin de diseñar políticas adecuadas de intervención pedagógica que garanticen la efectividad del proceso de enseñanza -aprendizaje, con el fin de garantizar aprehensión de los conocimientos buscando el desempeño efectivo de los estudiantes lo que implica una correcta y adecuada aplicación de las prácticas pedagógicas de los docentes.

Según cifras ofrecidas por el portal SPADIES a diciembre 2012 el índice de deserción en educación superior en el departamento Norte de Santander alcanzaba el $54,2 \%$ y en la Universidad Francisco de Paula Santander la tasa de deserción acumulada por cohorte era del $52,1 \%$ lo que se convierte en un problema de especial atención dado que al ser la UFPS una institución de educación de carácter público se debe garantizar el aprovechamiento de los recursos destinados por el gobierno nacional, por ello todas la acciones que se desarrollen en procura de disminuir dichos índices de deserción son bien recibidas.

Las investigaciones anteriormente mencionadas en neuropsicología, neurofisiología y en la psicología indican resultados como nuevos enfoques sobre cómo los seres humanos aprenden. Lo expuesto anteriormente sirve como fuente motivadora para el desarrollo de esta propuesta investigativa la cual toma como muestra a los estudiantes del programa académico de Licenciatura en Matemáticas de la Facultad de Educación, Artes y Humanidades de la UFPS, en los que se analiza la in- cidencia de los Estilos de Aprendizaje representados en el modelo de Honey y Mumford, con base a la Teoría de Kold quien propuso cuatro estilos de aprendizaje (Alonso, 1994 pág.104) que son, activo, reflexivo, pragmático, teórico.

El objetivo que se buscaba a través de esta investigación fue el identificar los estilos de aprendizaje presentes en los estudiantes del programa académico de Licenciatura en Matemáticas, bajo la óptica del modelo de Kold, y así poder determinar la existencia de la posible relación entre los diversos estilos que establece esta teoría y el modalidad de educación pública o privada del cual es egresado de bachiller.

\section{METODOLOGÍA}

Es un estudio de modalidad cuantitativo, bajo un diseño investigativo correlacional puesto que pretende determinar el grado de relación existente entre las variables susceptibles de medición (Hernández, 1991), las variables principales en esta investigación son los Estilos de Aprendizaje, del modelo de David Kold y el estrato social. Así mismo la investigación es no experimental ya que no se manipulara variable alguna. Para llegar a determinar la posible relación entre los estilos se hace necesario primero realizar un análisis descriptivo de los datos recolectados. Este estudio como resultado final da lugar a usos posteriores para la adecuación de las prácticas pedagógicas del docente y construcción de micro-currículos de las asignaturas que conforman el pensum académico del programa de licenciatura.

Participantes. Se trabajó con el total de los estudiantes matriculados en el programa de Licenciatura en Matemáticas de la UFPS durante el primer semestre del 2014, que totalizaban 45 estudiantes, distribuidos en 25 mujeres y 20 hombres, con edades entre los 15 y 35 años. Egresados en su mayoría de instituciones educación medía de carácter público del área metropolitana de Cúcuta y provenientes de los estratos socioeconómicos $1,2,3$. 
Instrumento. El instrumento aplicado está conformado por unos grupos de variables, inicialmente aquellas variables de modalidad socio-demográfico tales como: edad, género, modalidad del colegio, entre otras. Seguidas de las variables de proceso, las cuales se componen de las respuestas del Cuestionario de Honey y Alonso de Estilos de Aprendizaje (CHAEA): con 80 ítems, los cuales se agrupan en 4 grupos de 20 ítems; estructurados de manera estratégica para la identificación de los estilos de aprendizaje.

La clasificación de las respuestas del cuestionario CHAEA permite identificar los estilos de aprendizaje de cada uno de los estudiantes encuestados. Vale la pena resaltar que el instrumento utilizado para la caracterización de los estilos de aprendizaje es de amplio reconocimiento académico lo que garantiza su validez y confiabilidad.

\section{RESULTADOS Y DISCUSIÓN}

A continuación se presenta una breve reseña de las principales características de la población objeto de estudio en función de las variables analizadas. En cuanto a la edad de los estudiantes se puede afirmar que el $78 \%$ de se ubican en el rango entre 15 y 20 años, el $18 \%$ entre los 21 y 25 años y el porcentaje restante superan los 25 años. El $85 \%$ de ellos han egresado de instituciones de carácter público y el resto de instituciones privadas. En cuanto al género de los estudiantes, existe predominio del género femenino en un $56 \%$. En cuanto al estrato socio-económico del que provienen los estudiantes se encontró que el $36 \%$ son de estrato 1 , el $47 \%$ de estrato
2 y el $15 \%$ es de estrato 3. El $29 \%$ están matriculados en primer semestre, el $33 \%$ en segundo semestre, el $22 \%$ en tercer semestre y el $15 \%$ restante en el cuarto semestre.

Como resumen de lo mencionado anteriormente, se puede afirmar que en el programa académico de Licenciatura en Matemáticas se matriculan más mujeres que hombres, con edades promedio entre 15 - 20 años, quienes en su mayoría son egresados de colegios públicos, provenientes de los estratos socioeconómico 1 y 2 , y el programa de Licenciatura en Matemáticas tiene solo cuatro semestres en curso.

Para la Identificación de los estilos de aprendizaje de acuerdo al modelo de David Kolb, quien nos dice que por un lado, podemos partir:

a) de una experiencia directa y concreta: estudiante activo.

b) o, bien de una experiencia abstracta, que es la que tenemos cuando leemos acerca de algo o cuando alguien nos lo cuenta: estudiante teórico.

Las experiencias que tengamos, concretas o abstractas, se transforman en conocimiento cuando las elaboramos de alguna de estas dos formas:

a) reflexionando y pensando sobre ellas: estudiante reflexivo.

b) Experimentando de forma activa con la información recibida: estudiante pragmático.

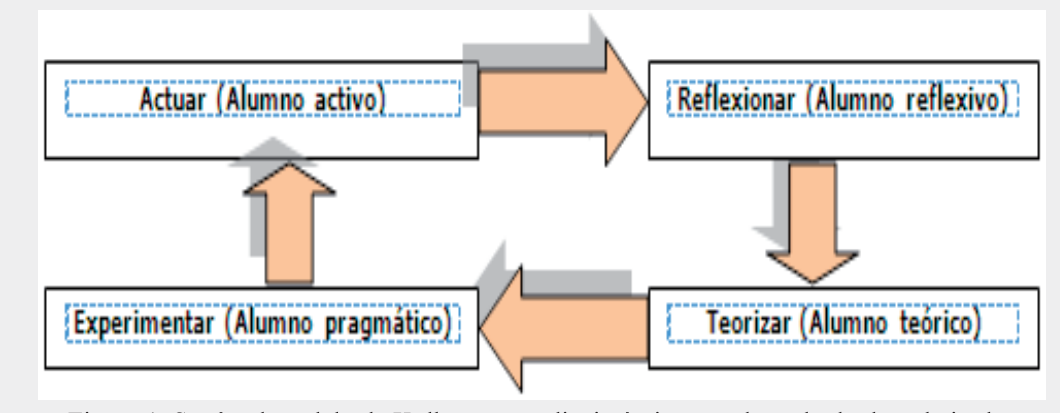

Figura 1. Según el modelo de Kolb un aprendizaje óptimo es el resultado de trabajar la información en cuatro fases: 
Eco.Mat

Cúcuta-Colombia

Vol. 6

No. 1

Enero-Diciembre 2015

ISSN 1794-8231

ISSN 2462 - $8794 \mathrm{We}$ PP: $69-77$
Estudio correlacional entre la modalidad de educación pública o privada y el estilo de aprendizaje

En la práctica, la mayoría de nosotros tendemos a especializarnos en una, o como mucho en dos, de esas cuatro fases, por lo que se pueden diferenciar cuatro modalidades de estudiantes, dependiendo de la fase en la que prefieran trabajar:

- Estudiante activo.

- Estudiante reflexivo.

- Estudiante teórico.

- Estudiante pragmático.

De acuerdo a la información obtenida de la recolección de los datos, se puede afirmar que el $57 \%$ de los estudiantes del programa de Licenciatura en Matemáticas son reflexivos de los cuales el $37 \%$ son mujeres y el $20 \%$ son hombres, quienes tienden a adoptar la postura de un observador que analiza sus experiencias desde muchas perspectivas distintas.

Recogen datos y los analizan detalladamente antes de llegar a una conclusión. Para ellos lo más importante es ese acopio de datos y su análisis concienzudo, así que procuran posponer las conclusiones todo lo que pueden. Son precavidos y analizan todas las implicaciones de cualquier acción antes de ponerse en movimiento. En las reuniones observan y escuchan antes de hablar procurando pasar desapercibidos. La pregunta que quieren responder con el aprendizaje es ¿por qué...?

Seguidamente se determinó que el 34\% de los estudiantes encuestados son pragmáticos, distribuidos en igual porcentaje en cada género. Les gusta probar ideas, teorías y técnicas nuevas, y comprobar si funcionan en la práctica. Les gusta buscar ideas y ponerlas en práctica inmediatamente, les aburren e impacientan las largas discusiones sobre la misma idea de forma interminable. Son básicamente gente práctica, apegada a la realidad, a la que le gusta tomar decisiones y resolver problemas. Los problemas son un desafío y siempre están buscando una manera mejor de hacer las cosas. La pregunta que quieren responder con el aprendizaje es ¿qué pasaría si...?

Los estudiantes pragmáticos aprenden mejor con actividades que relacionen la teoría y la práctica, cuando ven a los demás hacer algo, cuando tienen la posibilidad de poner en práctica inmediatamente lo que han aprendido.

Les cuesta más trabajo aprender cuando lo que aprenden no se relaciona con sus necesidades inmediatas, con aquellas actividades que no tienen una finalidad aparente o cuando lo que hacen no está relacionado con la "realidad".

Se determinó que el 15\% de los estudiantes encuestados son activos, de los cuales el $11 \%$ son mujeres y el $4 \%$ son hombres; que se involucran totalmente $y$ sin prejuicios en las experiencias nuevas. Disfrutan el momento presente y se dejan llevar por los acontecimientos. Suelen ser entusiastas ante lo nuevo y tienden a actuar primero y pensar después en las consecuencias. Llenan sus días de actividades y tan pronto disminuye el encanto de una de ellas se lanza a la siguiente. Les aburre ocuparse de planes a largo plazo y consolidar proyectos, les gusta trabajar rodeados de gente, pero siendo el centro de la actividades. La pregunta que quieren responder con el aprendizaje es ¿cómo?

Los estudiantes activos aprenden mejor cuando se lanzan a una actividad que les presente un desafío, cuando realizan actividades cortas de resultado inmediato o cuando hay emoción, drama y crisis.

Les cuesta más trabajo aprender cuando tienen que adoptar un papel pasivo, cuando tienen que asimilar, analizar e interpretar datos o cuando tienen que trabajar solos.

Por último, se determinó que el $12 \%$ de los Matemáticas son teóricos, distribuidos así, un $8 \%$ son mujeres y el $4 \%$ restante son hombres. Quienes adaptan e integran las observaciones estudiantes del programa de Licenciatura en 
que realizan en teorías complejas y bien fundamentadas lógicamente. Piensan de forma secuencial y paso a paso, integrando hechos dispares en teorías coherentes. Les gusta analizar y sintetizar la Información y su sistema de valores premia la lógica y la racionalidad. Se sienten incómodos con los juicios subjetivos, las técnicas de pensamiento lateral y las actividades faltas de lógica clara. La pregunta que quieren responder con el aprendizaje es ¿qué?
Los estudiantes teóricos aprenden mejor a partir de modelos, teorías, sistemas con ideas y conceptos que presenten un desafío o cuando tienen oportunidad de preguntar e indagar.

Les cuesta más trabajo aprender con actividades que impliquen ambigüedades incertidumbre, en situaciones que enfaticen las emociones y los sentimientos o cuando tienen que actuar sin un fundamento teórico.

Tabla I. FRECUENCIA DE LOS ESTRATOS SOCIALES

\begin{tabular}{cccc}
\hline Estrato Socioeconómico & Frecuencia & Porcentaje & Porcentaje acumulado \\
\hline Uno & 16 & 35,0 & 35,0 \\
Dos & 21 & 46,0 & 82,0 \\
Tres & 8 & 17,0 & 100,0 \\
Total & 45 & 100,0 & \\
\hline
\end{tabular}

La tabla muestra que el $46 \%$ de los estudiantes son de estrato dos y un $82 \%$ son de estrato 1 y 2 .

Tabla II. ESTRATO SOCIO ECONÓMICO POR SEMESTRE

\begin{tabular}{cccc}
\hline & \multicolumn{3}{c}{ Estrato Socioeconómico } \\
\cline { 2 - 4 } Semestre & Uno & Dos & Tres \\
\hline Primero & 5 & 4 & 4 \\
Segundo & 6 & 8 & 2 \\
Tercero & 3 & 5 & 1 \\
Cuarto & 2 & 4 & 1 \\
\hline
\end{tabular}

La tabla nos muestra que 8 estudiantes son de estrato dos y pertenecen a segundo semestre.

Tabla III. CRUCE ENTRE LOS ESTILOS DE APRENDIZAJE DESDE EL MÉTODO PROPUESTO POR DAVID KOLB.

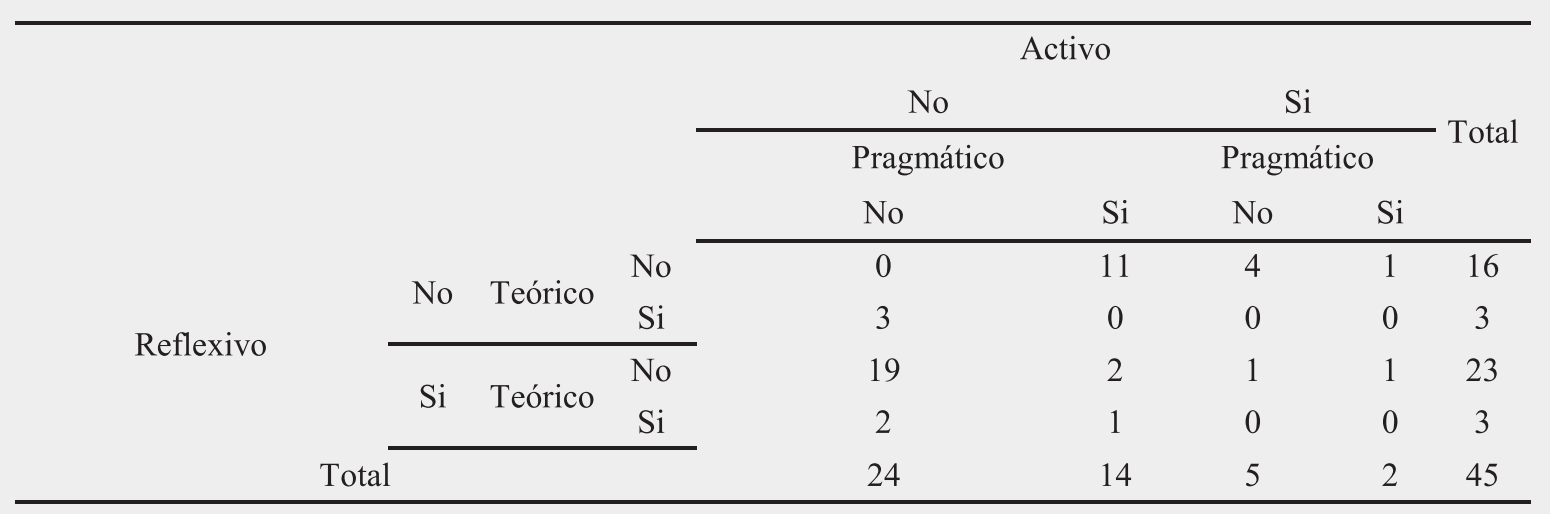


Eco.Mat

Cúcuta-Colombia

Vol. 6

No. 1

Enero-Diciembre 2015 ISSN 1794-8231

ISSN $2462-8794 \mathrm{Web}$ PP: $69-77$

Estudio correlacional entre la modalidad de educación pública o privada y el estilo de aprendizaje

Como se puede observar en la tabla anterior 7 estudiantes son activos, 16 son pragmáticos, 26 son reflexivos, 6 son teóricos, un estudiante es activo y pragmático, uno es activo y reflexivo, uno es activo y reflexivo, dos son pragmáticos y reflexivos, uno es activo, pragmático y reflexivo, uno es pragmático, reflexivo y teórico.

Tabla IV. ESTRATO SOCIOECONÓMICO VERSUS MODALIDAD DEL COLEGIO

\begin{tabular}{cccc}
\hline \multirow{2}{*}{ Estrato Socioeconómico } & \multicolumn{2}{c}{ Modalidad Del Colegio } \\
\cline { 2 - 3 } & Privado & Publico \\
\hline Uno & 3 & 13 \\
Dos & 3 & 18 \\
Tres & 1 & 7 \\
\hline
\end{tabular}

Como se observa en la tabla los estudiantes del programa de matemáticas, la gran mayoría son de colegios públicos.

Tabla V. ESTILOS DE APRENDIZAJE DE KOLB VERSUS MODALIDAD DEL COLEGIO

\begin{tabular}{ccc}
\hline \multirow{2}{*}{ Estilo de Aprendizaje } & \multicolumn{2}{c}{ Modalidad del Colegio } \\
\cline { 2 - 3 } & Privado & Publico \\
\hline Activo & 2 & 5 \\
Reflexivo & 1 & 25 \\
Teórico & 0 & 6 \\
Pragmático & 4 & 12 \\
\hline
\end{tabular}

Se observa que los estilo de aprendizaje es en gran porcentaje de colegios públicos vale resaltar que el mayor porcentaje del estilo de aprendizaje de la modalidad de colegio privado es el pragmático.

Tabla VI. CRUCE ENTRE ESTILOS DE APRENDIZAJE CON ESTRATO SOCIOECONÓMICO Y SEMESTRE ESTUDIANTIL.

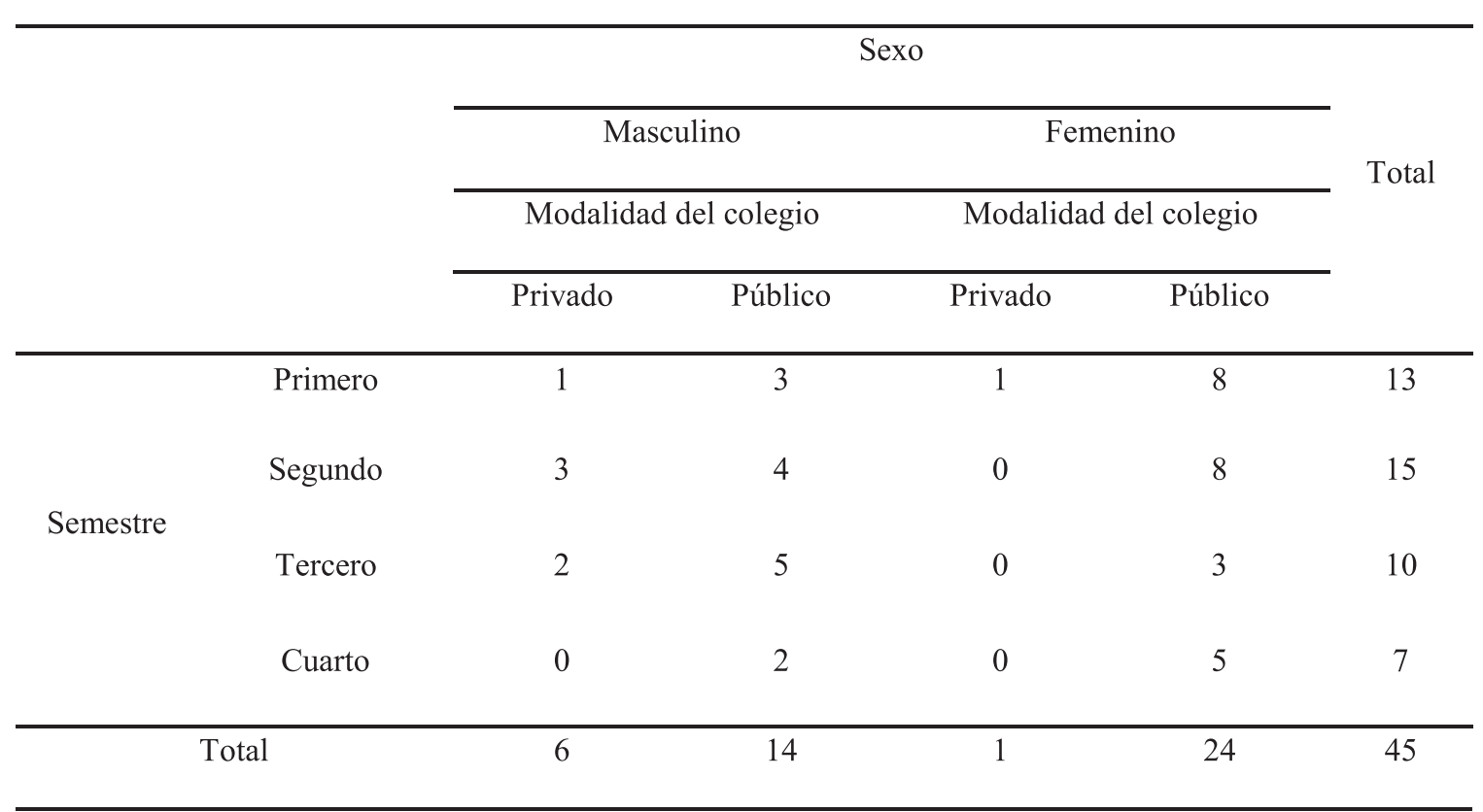


Se observa la relación existente entre los estilos de aprendizaje de Kolb y el estrato socioeconómico de los estudiantes del programa de matemáticas, nos dice que en el primer semestre hay 13 estudiantes de los cuales 4 hombres, 1 egresado de colegio privado, 3 de colegio público, y 9 mujeres 1 egresadas de colegio privado, 8 de colegio público, en el segundo semestre hay 15 estudiantes de los cuales 7 hombres, 3 egresado de colegio privado, 4 de colegio público, y 8 mujeres egresadas de colegio público, en el tercer semestre hay 10 estudiantes de los cuales 7 hombres, 2 egresado de colegio privado, 5 de colegio público, y 3 mujeres egresadas de colegio público y por último en el cuarto semestre hay 7 estudiantes de los cuales 2 hombres egresado colegio público, y 5 mujeres egresadas de colegio público .

Una vez categorizados los estudiantes en los diversos estilos de aprendizaje según el modelo teórico en estudio, surgió la inquietud en analizar si existía algún modalidad de relación entre cada estilo del modelo, y el modalidad de colegio de egresado de bachiller referente a si es público o privado, es por ello que se procedió a realizar la prueba de independencia Chi-cuadrado, la cual permite determinar si existe una relación entre dos variables categóricas. Es necesario resaltar que esta prueba nos indica si existe o no una relación entre las variables, pero no indica el grado o el modalidad de relación; es decir, no indica el porcentaje de influencia de una variable sobre la otra o la variable que causa la influencia. La prueba de independencia del Chi-cuadrado, parte de la hipótesis que las variables en consideración son independientes. A continuación se presentan los resultados asociados a esta prueba.

Tabla VII. PRUEBA DE INDEPENDENCIA

\begin{tabular}{|c|c|c|c|}
\hline \multicolumn{4}{|c|}{ Prueba Chi-Cuadrado de Pearson } \\
\hline & Activo & Reflexivo & Teórico \\
\hline Reflexivo & 0,089 & & \\
\hline Teórico & 0,259 & 0,679 & \\
\hline Pragmático & $0,001 *$ & $0,001 *$ & 0,299 \\
\hline
\end{tabular}

* El nivel de significancia $\alpha$ es menor que 0,05

\begin{tabular}{llrrrr}
\multicolumn{7}{c}{ Tabla VIII. PRUEBAS CHI-CUADRADO DE PEARSON } & \\
& & ACTIVO & REFLEXIVO & TEORICO & PRAGMÁTICO \\
\hline Modalidad del Colegio & Chi-cuadrado & 1,069 & 6,000 & 1,000 & 1,000 \\
& gl & 1 & 1 & 1 & 1 \\
& Sig. &, $000^{\mathrm{a}}$ &, $011^{\mathrm{a},{ }^{*}}$ &, $000^{\mathrm{a}, \mathrm{c}}$ &, $000^{\mathrm{a}}$ \\
\hline
\end{tabular}

La prueba de independencia Chi-cuadrado, permite determinar si existe una relación entre dos variables categóricas. Es necesario resaltar que esta prueba nos indica si existe o no una relación entre las variables, pero no indica el grado o el modalidad de relación; es decir, no indica el porcentaje de influencia de una variable sobre la otra o la variable que causa la influencia. La prueba de independencia del Chi-cuadrado, parte de la hipótesis que las variables en consideración son independientes.
Enero-Diciembre 2015 ISSN 1794-8231 ISSN 2462 - $8794 \mathrm{Web}$ PP: $69-77$ 
Eco.Mat

Cúcuta-Colombia

Vol. 6

No. 1

Enero-Diciembre 2015 ISSN 1794-8231

ISSN 2462 - $8794 \mathrm{We}$ PP: $69-77$
Estudio correlacional entre la modalidad de educación pública o privada y el estilo de aprendizaje

La prueba chi-cuadrado indica que existe relación entre los estilos pragmáticos activos y pragmáticos reflexivos, la modalidad del colegio presenta relación con el estilo de aprendizaje Reflexivo del modelo de Kolb.

Tabla IX. PRUEBA DE CORRELACIÓN

Coeficiente Rho de Spearman

\begin{tabular}{lllll}
\hline & Activo & Reflexivo & Teórico & Pragmático \\
Reflexivo & $-0,254$ & & & \\
Teórico & $-0,168$ & $-0,062$ & & \\
Pragmático & $-0,063$ & $-0,493^{* *}$ & -0.155 & 0,000 \\
& & & & \\
Modalidad del Colegio & 0,000 & $0,010^{*}$ & 0,000 & \\
\hline
\end{tabular}

** La correlación es significativa en el nivel del 1\% ( 2 colas)

* La correlación es significativa en el nivel del 5\% ( 2 colas)
La prueba de correlación de Spearman, permite determinar si existe una relación entre dos variables categóricas preferiblemente ordinales. Es necesario resaltar que esta prueba nos indica la modalidad de correlación que existe entre las dos variables, su rango varía entre $[-1,1]$. Con valores cercanos a los límites inferior y superior del intervalo indica la presencia de una fuerte correlación negativa o positiva entre las variables, respectivamente.

La prueba de correlación de Spearman, corrobora la prueba de independencia en que existe relación entre la Modalidad del Colegio y el estilo de aprendizaje Reflexivo, e indica una relación moderada entre los estilos reflexivo y pragmático.

\section{CONCLUSIONES Y RECOMENDACIONES}

Se puede afirmar a partir del análisis de los resultados obtenidos de los estudiantes de Licenciatura en Matemáticas que los estilos de aprendizaje son muy diversos puesto que se encontró estudiantes ubicados en cada clasificación derivada del método, situación que lleva al docente a un mayor nivel de exigencia en cuanto a la planificación y diseño de actividades pedagógicas con el fin de garantizar la plena aprehensión de los conocimientos impartidos desde su asignatura.

Considerando los estilos de aprendizaje según el Modelo de Kolb se pudo observar en los estudiantes del programa de Licenciatura en Matemáticas que existe predominio del estilo reflexivo con un 58\%, seguido por el pragmático observable en un 35\%, los activos con un $16 \%$ y por último el teórico con un $13 \%$; en los estilos reflexivo, pragmático y teórico las mujeres fueron predominantes y en el activo se distribuyó equitativamente por géneros. Vale la pena resaltar que el $22 \%$ de los estudiantes encuestados poseen más de un estilo de aprendizaje según David Kolb. 
En lo que respecta a la correlación entre los diversos estilos de aprendizaje del modelo se puede concluir la relación existente entre los estilos Pragmático y Reflexivo.

Se puede concluir que los estilos, más predominante entre los estudiantes del programa de Licenciatura en Matemáticas es el Reflexivo, seguida del Pragmático y por último el Activo y Teórico Respectivamente.

El estudio confirmo que el $46 \%$ de los estudiantes son de estrato dos y un $82 \%$ son de estrato 1 y 2.

El $55 \%$ de los estudiantes son de sexo femenino las cuales en su mayoría son de estilo de aprendizaje Reflexivo.

Por último se resalta según la prueba de independencia de chi-cuadrado de Pearson y la prueba de correlación de Spearman indica que existe relación entre los estilos pragmáticos activos y pragmáticos reflexivos, y una relación entre la Modalidad del Colegio y el estilo de aprendizaje Reflexivo del modelo de Kolb, llegando a la conclusión que no importa la modalidad del colegio los estudiantes de Licenciatura en Matemáticas Tienen un estilo de aprendizaje Reflexivo.

\section{REFERENCIAS}

1. Alonso et al. (1994: pág. 104). Características de cada estilo según Alonso C, Domingo J, Honey P (1994), "Los estilos de aprendizaje: procedimientos de diagnóstico y mejora", Ediciones Mensajero, Bilbao, pp. 104-116.

2. Cazau Pablo (2001), Estilos de aprendizaje http://www.galeon.com/pcazau/guia esti01.htm (consultado en enero 2002)

3. Chalvin Marie Joseph (1995), "Los dos cerebros en el aula", TEA Ediciones, Madrid, 1995.

4. Chavero Blanco (2002). Hipermedia en Educación. El modo escritor como catalizador del proceso enseñanzaaprendizaje en la Enseñanza Secundaria
Obligatoria http://med.unex.es/Docs/ TesisChavero/Indice.html (consultado en septiembre 2002)

5. Chevrier Jacques (2001) Le style d'apprentissage : une perspective historique. http://www.acelf.ca/revue/ XXVIII/articles/02-chevrier.html\#h-7 (consultado en enero 2001)

6. Curry (1987). Integrating concepts of cognitive or learning style: A review with attention to psychometric standards. Otawwa, ON: Canadian College of Health Service Executives.

7. Dunn, R., Dunn, K. And Price, G. (1985) Manual: Learning Style Inventory. Lawrence, KS: Price Systems.

8. Eric Jenssen (1994) Unlocking The Code: Learning Styles. Brain Based Learning and teaching. USA : Turning Point Publisher; 1994

9. Estilos de aprendizaje y perspectivas de enseñanza. Descargado de: http://www. monografias.com/trabajos $12 /$ losestils/ losestils.shtml-112K.

10. Felder R. (1996), Matters of Style. ASEE Prism, 6(4), 18-23 (December 1996).

11. Garza, R. y Leventhal S. (2000) Aprender como Aprender. Mexico: Trillas.

12. Hunt,( 1979), en Chevrier J., Fortin, G y otros, 2000.

13. Jenssen Eric. (1994). Unlocking the Code: Learning Styles. Brain Based Learning and teaching. USA: Turning Point Publisher.

14. Keefe James (1988) Aprendiendo Perfiles de Aprendizaje: manual de examinador, Reston, VA: Asociación Nacional de Principal de Escuela de Secundaria. 